\title{
ON THE AUTOMATICITY OF THE RESPIRATORY CENTERS OF THE CATFISH AND CRUCIAN CARP
}

\author{
TAKESI HUKUHARA AND HIROMASA OKADA* \\ Department of Physiology, Okayama University Medical School
}

The problem as to whether the respiratory centers of fishes are endowed with the ability to discharge with inherent rhythmicity or not seems not to have been extensively studied. We have not been able to find any paper except that of Adrian and Buytendijk (1) in this field of research. They studied this problem on the goldfish, and recorded slow potential changes with the respiratory rhythm from the isolated brain stem (optic lobes and medulla oblongata) of the animal. They are of opinion that this slow potential change reflects a slow development and decline of the excitatory state of the nerve cells in the respiratory centers, and that the rhythmic activity of the respiratory centers can occur in the entire absence of sensory impulses. Recently we have studied the problem related to the automaticity of the respiratory centers on the catfish as well as carassius auratus by means of methods which are different from those used by the authors described above; the results of the experiments are reported in the following:

\section{METHODS}

As experimental animals catfish (parasilurus asotus Linné) weighing 200-250 g. and crucian carp (carassius auratus Linné) weighing 100-150 g. were used. The animal was clamped in a prone position and put in a bath filled with water without immersing the upper surface of the skull. Tap water was continuously flowed into the bath, while an equal amount of water was siphoned out of it. When the gill movements ceased as a consequence of cutting the trigeminal nerves, an appropriate rubber tubing was inserted into the mouth cavity to introduce the tap water into it.

The cranial cavity of the animal was opened wide to expose the dorsal surface of the brain, as well as the brain nerves. The unipolar microelectrode which was used to detect action potentials from particular regions of the brain stem was similar to that used in the experiments concerned with the respiratory centers of mammals (3). The electrode was connected to the grid of the first stage of a five stage R-C coupled push-pull amplifier, the time constant of which was 0.2 seconds. A large external electrode which consisted of a silver plate $1 \times 3 \mathrm{~cm}$. was fixed on the wound surface of the skull and connected to the earth side of the amplifier. The output of the amplifier was connected to the vibrator

Received for publication July 13, 1956.

* 福原 武, 岡田博匡 
of A-type of Yokogawa's electromagnetic oscillograph. The amplifier was also connected to the cathode-ray tube and loud-speaker for inspectatory and auditory control. The microelectrode was operated by the stereotaxic micromanipulator.

The abduction and adduction of the gill covers served as an index of the respiratory movements and were recorded by means of the electric manometer.

\section{RESULTS}

\section{Experiments on the brain stem of the catfish}

After the brain had been exposed, the olfactory lobes and tracts, forebrain, interbrain, the bulging part of the cerebellum (pars posterior cerebelli), and a greater part of the optic lobes, except for the caudal part lying near the cerebellum, were successively removed without producing any remarkable changes in the movements of the gill covers. When the brain was then transected at the level of the region of the medulla oblongata, in which the hypoglossal nerves originate, the movements of the gill covers ceased only transitorily. Lastly, all the brain nerves III-X, except the unilateral trigeminal nerve, were successively severed; owing to this procedure the movements of the gill covers ceased completely. Several minutes afterwards, however, the unilateral gill cover, the motor nerve of which was retained intact, moved again rhythmically; the movements were at first weak and became gradually stronger.

As to the localization of the respiratory centers of the fish the present knowledge seems not to be exact. Vulpian (1866) (5) said that "le noeud vital" is situated at the level of the under border of the cerebellum, and Steiner (1888) (4) said that the respiratory centers are, in squalius cephalus, situated in the lobes impar. With respect to this problem our experimental results concerned with the localization of the respiratory centers of mammals (3) were suggestive, and by analogical inference the microelectrode was dorsoventrally inserted into the region of the boundary of the acoustic tubercle and the facial lobe. The region is marked with the white circle $\mathrm{S}$ in fig. 1 . The results were that volleys

FIG. 1. Dorsal view of the brain of the catfish.

$\mathrm{S}$ marks the region into which the microelectrode was inserted.

F. B.: forebrain. M. B.: midbrain. Met. ceph.: metencephalon. Tub. acus.: tuberculum acusticae. Lob. fac.: lobus facialis. Med. oblon.: medulla oblongata. Tract. olf.: tractus olfactorius. Tract opt.: tractus opticus. N.L.l.: nervus linae lateralis.

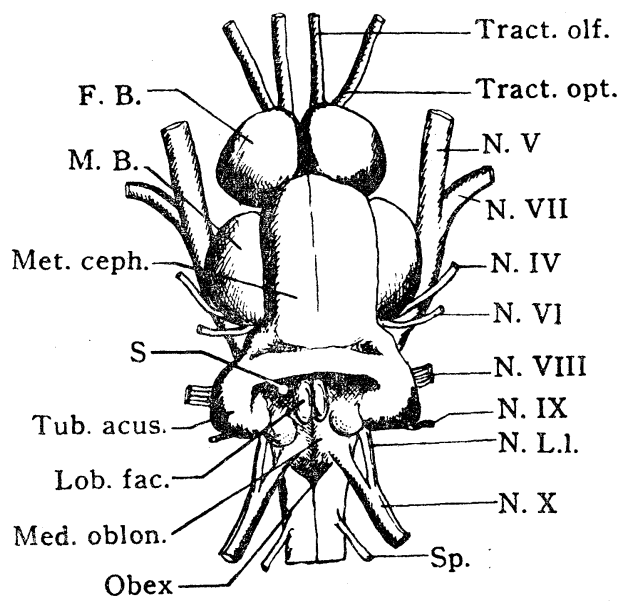


recurring rhythmically and consisting of spike discharges were detected, when the tip of the electrode reached the depth of $1.5-2.0 \mathrm{~mm}$., which was half of the dorsoventral diameter of that region of the brain stem. The rhythm of the volleys was the same as that of the movements of the gill cover, and two kinds of volleys could be observed: the one was produced simultaneously with the adduction of the gill cover, the other with the abduction; and the former was produced more frequently than the latter. Both adductory and abductory neurons were intermingled together in the region concerned. As regards the pattern of discharges, a trapezoidal pattern (3) was most frequently observed in adductory and abductory neurons, while a rectangular or steady pattern was observed only in rare cases. The examples are shown in figs. 2 and 3. In the former the volleys were produced during the abduction of the right gill cover, recurring with the rhythm of 47 per minute and consisting of 37 spike discharges, the frequency of which was 67 per second at the stage of constant frequency of the volley. In the latter the volleys were produced during the adduction of the gill cover concerned, and they were detected by varying the depth of the tip of the electrode, when about 30 minutes had elapsed after the detection of the volleys shown in fig. 2 . The volleys recurred with the rhythm of 72 per minute and consisted of 22 spike discharges, the frequency of which was 60 per second at the stage of constant frequency of the volley.

After the volleys described above had been recorded, the electrode was drawn out and the trigeminal nerve which had been left intact, was cut at its root. The movements of the gill cover then instantaneously stopped. The brain stem was now completely isolated from the rest of the body. The electrode was then again inserted into the same region as before; the volleys were in general

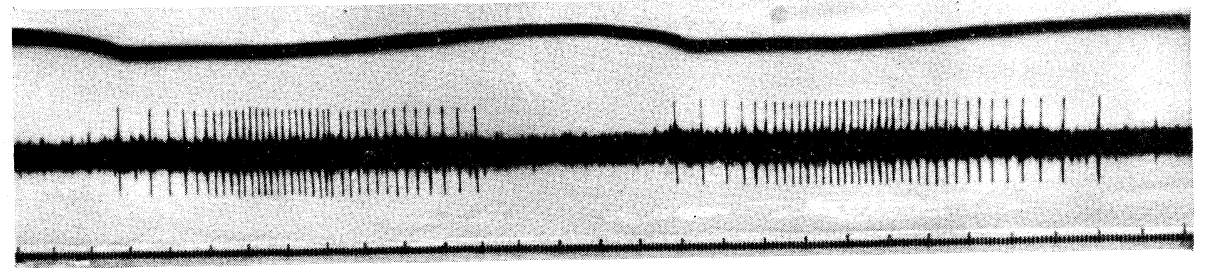

FIG. 2. Respiratory action potentials detected from the brain stem of the catfish. Body weight of the animal: $203 \mathrm{~g}$. Temperature of the water in the bath: $14^{\circ} \mathrm{C}$. Room temperature: $20^{\circ} \mathrm{C}$. Upper trace: mechanogram of the right gill cover. Adduction upwards. Lower trace: action potentials. Time marks 1/120 sec. (small dot).

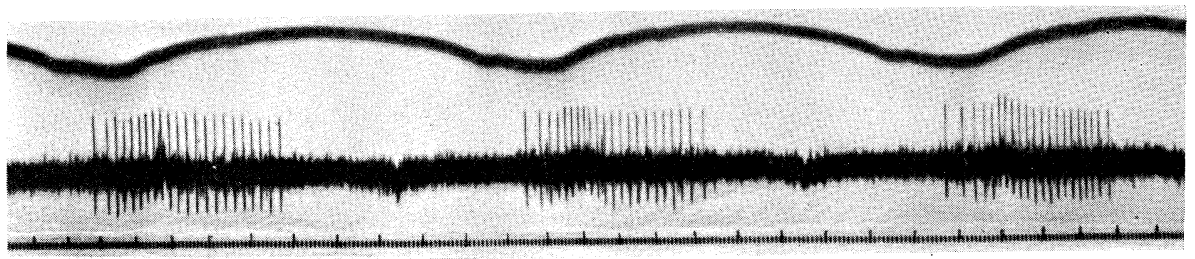

FIG. 3. Respiratory action potentials detected from the brain stem of the catfish. The same animal as that in fig. 2. Explanations are the same as in fig. 2. 


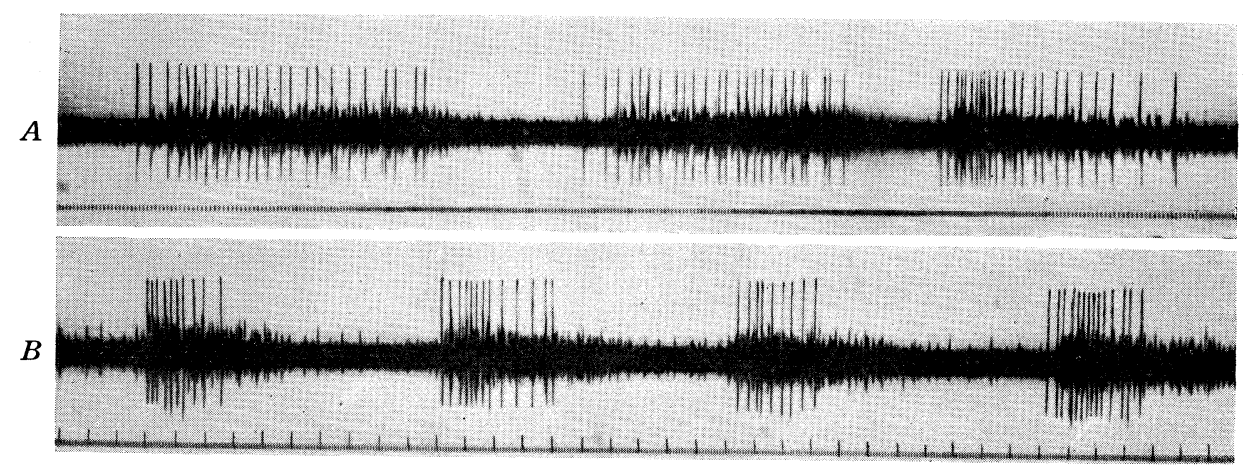

FIG. 4. Action potentials detected from the brain stem of the catfish isolated from the rest of the body.

Animal was the same as that in fig. 2 and 3.

$A$ and $B$ show the potentials detected respectively 3 and 5 minutes after the complete isolation of the brain stem.

detected by several trials, and in rare cases only by one trial. The example is shown in fig. $4 \mathrm{~A}$. The record was obtained 3 minutes after the isolation of the brain stem. The volleys recurred with the rhythm of 54 per minute. The frequency of these volleys was at the stage of constant frequency 50 per second and the number of spikes was $21-25$ in a volley. Two minutes afterwards, as shown in fig. $4 B$, the duration of the volleys was remarkably shortened $(0.21-$ $0.30 \mathrm{sec}$.) and the rhythm of the volleys was accelerated (70 per minute), although the number of spikes in a volley was decresed (9-14). On the whole, the behavior of the volleys was roughly similar to that observed immediately before the isolation of the brain stem. Soon thereafter the decline of the discharge occurred and became more and more pronounced with the elapse of time; both the number and frequency of spikes in a volley were gradually decreased, and the rhythm of the volley became gradually slow and irregular, although the volleys, which were increased in number and frequency of spikes, were only occasionally interpolated. About 30 minutes after the isolation of the brain stem, volleys consisting of only several spikes were produced with a slow and irregular rhythm. Such a transient survival of the isolated brain stem would be due to the deteriolation of that region, which was brought about by circulatory and respiratory failure. Similar results were obtained in 5 cases.

The volleys were also detected from the region described above in the brain stem removed out of the cranial cavity. In these cases, the volleys were irregularly produced and their behavior was similar to that observed late in the desclining stage of the discharge detected from the brain stem isolated and left in the cranial cavity.

From the results described above it is highly probable that the volleys were detected from the respiratory centers or from their centrifual pathways, and that the centers are endowed with the automaticity.

LOCALIZATION OF MICROELECTRODE SITE

The localization of the microelectrode site was histologically examined in 
one experiment, the results of which are shown in figs. 2, 3 and 4 . The brain stem was fixed with formalin, embedded in paraffin, transected serially in thickness of 30 micra and stained by Nissl's method.

In the section of the brain stem* transected at the level of circle $\mathrm{S}$ (see fig. 1), the trace of the electrode was perceptible as a tissue defect filled with blood (fig. 5). Judging from this trace the electrode had been inserted at the medial border of the cerebellar crest dorsoventrad, till its tip reached the region situated just dorsomedially to the spinal tract of the trigeminal nerve, as well as dorsally to the substantia gelatinosa Rolandi. The nerve fibers, of which the acoustico-lateral decussation consisted, passed through the region concerned, and the small nerve cells lay scattered among the fibers. The results described above suggest that the respiratory impulses might be produced in the small cell group lying scattered in the acoustico-lateral decussation, or detected from the centrifugal pathways of the trigeminal nucleus. As regards the precise localization of the respiratory centers of the catfish, it remains to be solved.

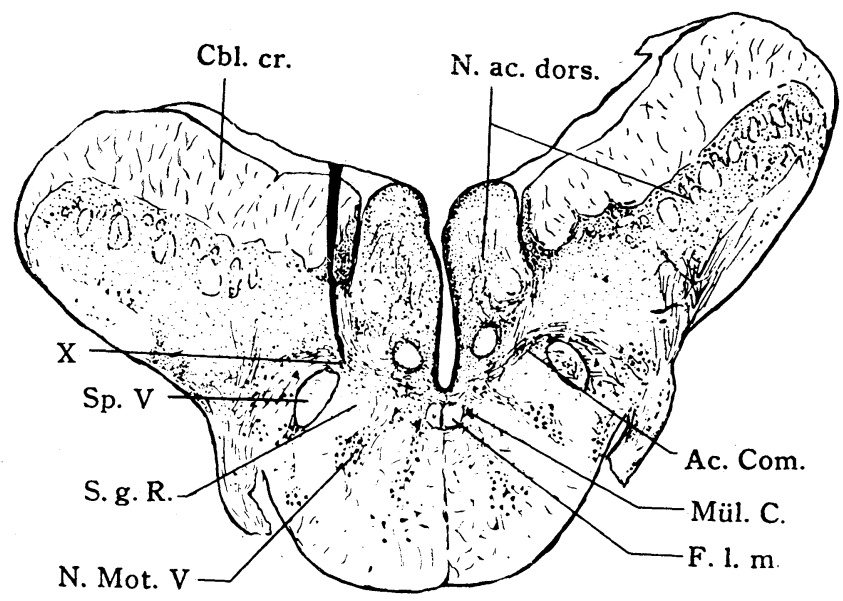

FIG. 5. Point of detection of the respiratory potentials in the brain stem of the catfish (section at the level of $S$ in fig. 1 ).

$\mathrm{X}$ shows the trace of the tip of the microelectrode. Cbl. cr.: cerebellar crest. N. ac. dors.: nucleus acousticolateralis dorsalis. Sp. V: radix spinalis nervi trigemini. S. g. R.: substantia gelatinosa Rolandi. N. Mot. V.: nucleus motorius trigemini. Ac. Com.: acousticolateral decussation. Mül. C.: Müller's cell. F. 1. m.: fasciculus longitudinalis medialis.

\section{Experiments on the brain stem of crucian carp}

The results obtained in the experiments ( 5 cases) on the crucian carp were similar to those in the catfish. After the brain stem had been exposed as shown in fig. $6 \mathrm{~A}$, the pars posterior cerebelli was turned up rostrad and transected at its base, and the vagal lobes were pushed aside to expose the lobus impar as

* The terms used to denote the regions of the brain stem are based upon those used by Bartelmez in his report (2). 
FIG. 6. Dorsal view of the brain of the crucian carp.

$A$ : Unimpaired brain. $B$ : Pars posterior was removed and vagal lobes were pushed aside. F. B.: forebrain. Lob. opt.: lobus opticus. Cerebel.: Cerebellum. Lob. imp.: lobus impar. Lob. vagi: lobus vagi. S: Point of insertion of the microelectrode.

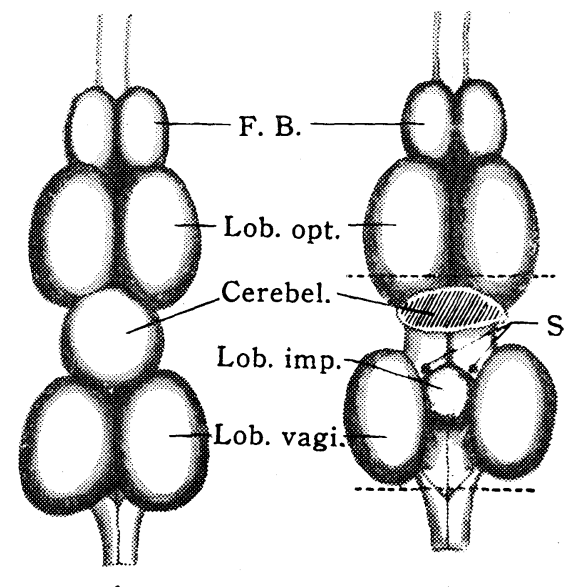

A

B

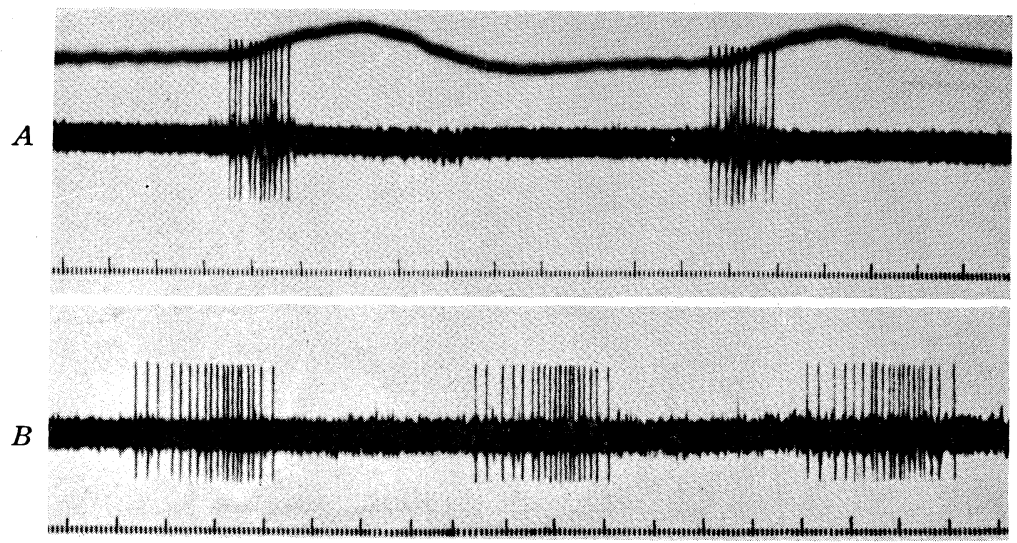

FIG. 7. Action potentials detected from the brain stem of the crucian carp.

A. Body weight.: $115 \mathrm{~g}$. Temperature of the water in the bath: $20^{\circ} \mathrm{C}$. Room temperature: $32^{\circ} \mathrm{C}$.

Volleys were produced simultaneously with the adduction of the gill covers.

$B$. Body weight: $150 \mathrm{~g}$. Temperature of the water in the bath: $23^{\circ} \mathrm{C}$. Room temperature: $31^{\circ} \mathrm{C}$.

Volleys were produced simultaneously with the abduction of the gill covers.

shown in fig. $6 B$. The microelectrode was then inserted dorsoventrally into the region (marked with $\mathrm{S}$ in fig. $6 B$ ) which was situated rostrolaterad to the lobus impar. When the tip of the electrode reached the depth of 1.5-2 mm., volleys were detected, and the rhythm of the volleys was the same as that of the movements of the gill covers. Two kinds of volleys were classified as in the case of catfish: the one was produced simultaneously with the adduction of the gill covers, the other with their abduction; and the former appeared more often than the latter. The patterns of impulse discharge were similar to those in the 
catfish. The examples were shown in fig. 7. In fig. $7 \mathrm{~A}$ the volleys recurring with the rhythm of 72 in a minute were produced from a single adductory neuron. The frequency of impulses in a volley was 120 per second, In fig. $7 B$ the volleys recurring with the rhythm of 103 in a minute were produced from a single abductory neuron, and the impulse frequency was at the stage of constant frequency 111 per second.

After the action potentials described above had been recorded, the electrode was drawn out, the region of the brain situated rostrad to the cerebellum was removed, all the brain nerves were cut, and the medulla oblongata was transected at its caudal end. The result was that the movements of the gill covers ceased completely. The electrode was then again inserted into the region mentioned above, In successful cases the volleys were detected about several minutes after the manipulation of the electrode. The examples are shown in fig. 8. In this case the volleys consisting of 12 spikes, the frequency of which was 22 per second, were produced rhythmically and recurrently, although volleys which were increased in number and decreased in frequency (fig. $8 \mathrm{~B}$ ) were occasionally interpolated. The number and frequency were always smaller than those before the isolation of the brain stem.

With the elapse of time, the ability of the brain stem discharging impulses fell gradually into decay, till at last it was completely abolished about 20 minutes after the isolation of the brain stem. In another 4 cases similar results were obtained.

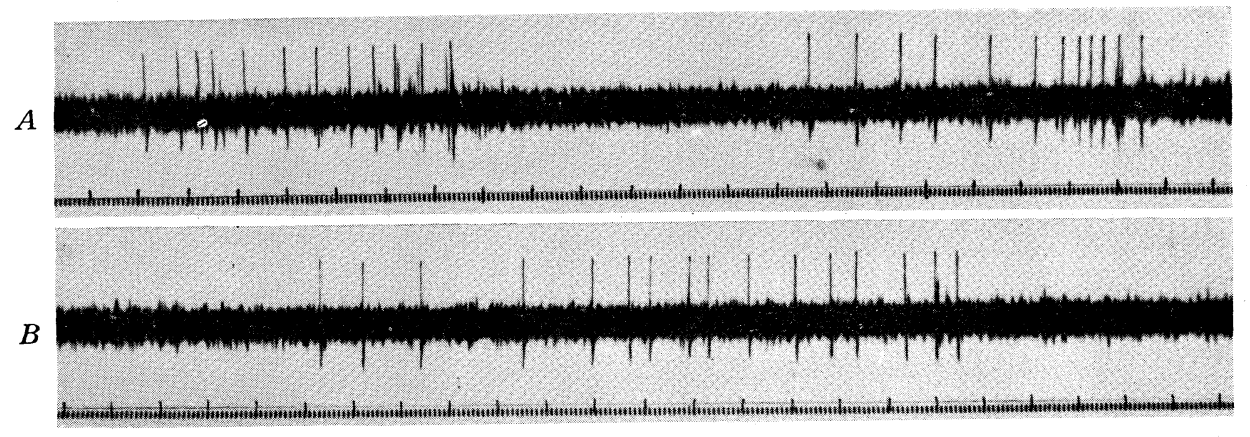

FIG. 8. Action potentials detected from the isolated brain stem of the crucian carp.

Body weight: $150 \mathrm{~g}$. Temperature of the water in the bath: $14^{\circ} \mathrm{C}$. Room temperature: $20^{\circ} \mathrm{C}$.

$A$ shows the volleys detected 5 minutes after the complete isolation of the brain stem.

$B$ shows a long lasting volley interpolated. Explanations see text.

SUMMARY

By means of a microelectrode technique the experiments were performed to contribute to the solution of the question on the localization, as well as on the automaticity, of the respiratory centers in the catfish and the crucian carp. The results are summarized as follow: 
1) When at the region of the boundary of the acoustic tubercle and the facial lobe in the catfish, and at the region situated rostro-laterad to the lobus impar in the crucian carp, the microelectrode was inserted dorsoventrally half of the length of the dorso-ventral diameter of that region, the volleys consisting of spike discharges were detected recurrently with the rhythm of the movements of the gill covers.

2) The histologic studies revealed that the tip of the electrode reached the region situated dorsad to the substantia gelatinosa Rolandi which was located dorsad to the motor trigeminal nucleus.

3 ) In the brain stem which was isolated rostrad from the lobus opticus and caudad from the spinal cord, and in which all the brain nerves were cut, the volleys whose aspect was similar to that of the volleys observed before the isolation of the brain stem were detected from the region described in 2).

4) From the results described above, it was concluded that the respiratory centers of the catfish, as well as those of the crucian carp, are most probably endowed with the ability to discharge with inherent rhythmicity.

The expense of this research was covered by a grant from the Ministry of Education.

\section{REFERENCES}

1. Adrian, E. D. AND F. J. J. BuYtendiJK. Potential changes in the isolated brain stem of the goldfish. J. Plysiol. 17: 121, 1931.

2. Bartelmez, G. W. Mauthner's cell and the nucleus motorius tegment. J. comp. Neurol. 25: 87, 1915.

3. Hukuhara, T., S. Nakayama AND H. OKada. Action potentials in the normal respiratory centers and their centrifugal pathways in the medulla oblongata and spinal cord. Jap. J. Physiol. 4: 145, 1954.

4. Steiner, J. Die Functionen des Zentralnervensystems und ihre Phylogenese, II Abt. Fishe, S. 31. Braunschweig, 1881; Tiegerstedts Handb. d. physiol. Methodik, III, 2, 159, Leipzig, von S. Hirzel, 1911.

5. Vulpian, A. Physiologie générale et comparée du système nerveux, quoted from Wintersteins Handb. d. vergleich. Physiol. Bd. 1, 2. Hälfte, 647, Jena, Gustav Fischer, 1921. 